\title{
QUASISYMMETRIC KOEBE UNIFORMIZATION WITH WEAK METRIC DOUBLING MEASURES
}

\author{
KAI RAJALA AND MARTTI RASIMUS
}

\begin{abstract}
We give a characterization of metric spaces quasisymmetrically equivalent to a finitely connected circle domain. This result generalizes the uniformization of Ahlfors 2-regular spaces by Merenkov and Wildrick [8].
\end{abstract}

\section{INTRODUCTION}

A homeomorphism $f$ between metric spaces $(X, d)$ and $\left(Y, d^{\prime}\right)$ is quasisymmetric if there exists a homeomorphism $\eta:[0, \infty) \rightarrow[0, \infty)$ such that

$$
\frac{d^{\prime}(f(x), f(y))}{d^{\prime}(f(x), f(z))} \leqslant \eta\left(\frac{d(x, y)}{d(x, z)}\right)
$$

for all distinct points $x, y, z \in X$. Quasisymmetric maps form a natural generalization of conformal maps to the setting of abstract metric spaces. In particular, the uniformization problem for quasisymmetric maps is important due to applications in areas such as geometric group theory, complex dynamics, and geometric topology.

The uniformization problem asks which spaces admit quasisymmetric maps onto some standard space such as $\mathbb{S}^{2}$. Bonk and Kleiner [2] solved the problem for Ahlfors 2-regular spheres $(X, d)$, i.e., topological spheres for which the two-dimensional Hausdorff measure $\mathcal{H}_{d}^{2}$ satisfies

$$
C^{-1} r^{2} \leqslant \mathcal{H}_{d}^{2}\left(B_{d}(x, r)\right) \leqslant C r^{2} \text { for all } x \in X, 0<r<\operatorname{diam} X .
$$

Bonk and Kleiner showed that linear local connectedness (see Section 2) is a necessary and sufficient condition for 2-regular spheres to be quasisymmetrically equivalent to $\mathbb{S}^{2}$. Here and in what follows, we equip $\mathbb{S}^{2}$ with the standard spherical metric.

Later, Merenkov and Wildrick [8] considered the multiply connected setting, generalizing the classical Koebe uniformization. They gave a characterization for the finitely connected Ahlfors 2-regular surfaces that are quasisymmetrically equivalent to circle domains in $\mathbb{S}^{2}$, and generalized it to countably connected surfaces with some additional geometric properties. Here a circle domain is an open and connected set whose set of complementary components consists of disks and points.

Mathematics Subject Classification 2010: Primary 30L10, Secondary 30C65, $28 \mathrm{~A} 75$.

The authors were supported by the Academy of Finland, project number 308659 . 
We refer to [8] and the related work by Bonk [1] for further motivation and background.

Our aim is to find similar characterizations for surfaces that need not be 2-regular, such as fractal surfaces. Uniformization results for fractal surfaces are of great importance in view of applications, cf. [3], [8, Section 2], but one cannot expect results as strong as above to hold.

In [7], Lohvansuu and the authors introduced the weak metric doubling measures, generalizing the metric doubling measures, or Strong $A_{\infty}$-weights, of David and Semmes [4]. These are, roughly speaking, measures that can be used to construct quasisymmetric deformations for a given metric, see Section 2 for the precise definition. We then gave a version of the Bonk-Kleiner theorem in terms of the existence of such measures.

In this paper we apply the weak metric doubling measures to finitely connected surfaces. Namely, we have the following generalization of the characterization given by Merenkov and Wildrick.

THEOREM 1.1. Let $X$ be a metric space homeomorphic to a domain in $\mathbb{S}^{2}$ such that $\bar{X} \backslash X$ contains finitely many components. Then $X$ is quasisymmetrically equivalent to a circle domain if and only if it is linearly locally connected, carries a weak metric doubling measure and $\bar{X}$ is compact.

Here $\bar{X}$ is the completion of $X$. The "only if" part of Theorem 1.1 follows from the definitions in a straightforward manner. Theorem 2.2 below is a quantitative version of the "if" part.

To prove this, we first apply the weak metric doubling measure to suitably deform the metric on $X$. We show that the deformed space is reciprocal in the sense of [10], and therefore admits a quasiconformal map into $\mathbb{S}^{2}$ by a recent result of Ikonen [6]. We then apply geometric estimates to show that this map, when suitably normalized, is quasisymmetric. Some of these are related to the results in [1], see Section 6 below. Our approach is different from those in [8] and [7], both of which apply the Bonk-Kleiner theorem.

\section{WEAK METRIC DOUBLING MEASURES}

For $x, y \in X$ and $\delta>0$, a finite sequence of points $x_{0}, x_{1}, \ldots, x_{m}$ in $X$ is a $\delta$-chain from $x$ to $y$, if $x_{0}=x, x_{m}=y$ and $d\left(x_{j}, x_{j-1}\right) \leqslant \delta$ for every $j=1, \ldots, m$. Notice that in every connected metric space each pair of points can be connected by a $\delta$-chain for any $\delta>0$.

Recall that a measure $\mu$ in a metric space $(X, d)$ is doubling if there is $C_{D} \geqslant 1$ such that

$$
\mu\left(B_{d}(x, 2 r)\right) \leqslant C_{D} \mu\left(B_{d}(x, r)\right) \text { for all } x \in X, r>0 .
$$

From now on we assume that $\mu$ is a Radon measure in $X$ that is doubling with constant $C_{D}$. 
In what follows, we use notation

$$
B_{x y}=B_{d}(x, d(x, y)) \cup B_{d}(y, d(x, y)) .
$$

Given $\mu$ and a "dimension" $s>0$, we define the $\mu$-length $q_{\mu, s}$ of points $x, y \in X$ as follows: set

$$
q_{\mu, s}^{\delta}(x, y):=\inf \left\{\sum_{j=1}^{m} \mu\left(B_{x_{j} x_{j-1}}\right)^{1 / s}:\left(x_{j}\right)_{j=0}^{m} \text { is a } \delta \text {-chain from } x \text { to } y\right\}
$$

and

$$
q_{\mu, s}(x, y):=\limsup _{\delta \rightarrow 0} q_{\mu, s}^{\delta}(x, y) .
$$

Definition 2.1. We say that $\mu$ is a $C_{W}$-weak metric doubling measure, or $W M D M$, of dimension $s>0$ in $(X, d)$, if for all $x, y \in X$,

$$
\frac{1}{C_{W}} \mu\left(B_{x y}\right)^{1 / s} \leqslant q_{\mu, s}(x, y) .
$$

From now on we assume that $\mu$ is a $C_{W}$-WMDM of dimension 2, and we abbreviate $q=q_{\mu, 2}$. See [7] for examples and further discussion.

Weak metric doubling measures should be compared to the metric doubling measures of David and Semmes. They are essentially defined by requiring that in addition to (1) also the reverse inequality holds.

Given $\lambda \geqslant 1$, a metric space $(X, d)$ is $\lambda$-linearly locally connected, or $L L C$, if for any $x \in X$ and $r>0$,

(i) if $y, z \in B_{d}(x, r)$ then there exists a continuum $K \subset B_{d}(x, \lambda r)$ with $y, z \in K$, and

(ii) if $y, z \in X \backslash B_{d}(x, r)$ then there exists a continuum $K \subset X \backslash$ $B_{d}(x, r / \lambda)$ with $y, z \in K$.

From now on we assume that $(X, d)$ is $\lambda$-LLC and homeomorphic to a circle domain such that $\bar{X}$ is compact and $\bar{X} \backslash X$ contains $M<\infty$ components. We denote by $C_{X}$ the ratio of the diameter of $(X, d)$ to the minimum distance between the components of $\bar{X} \backslash X$. We are now ready to state the main result of this paper.

THEOREM 2.2. There is an $\eta$-quasisymmetric homeomorphism from $(X, d)$ onto a circle domain $\Omega \subset \mathbb{S}^{2}$, where $\eta$ depends only on $\lambda, C_{X}$, $C_{D}, C_{W}$, and $M$.

As pointed out in the introduction, Theorem 1.1 is a straightforward consequence of Theorem 2.2. We do not know if the dependence on the number of components $M$ and the constant $C_{X}$ is necessary in Theorem 2.2, and if it admits extensions to countably connected domains corresponding to [8, Theorem 1.4]. The rest of the paper is dedicated to the proof of Theorem 2.2. 


\section{Deformation of THE MEtric}

Theorem 2.2 is proved by showing that the $\mu$-length $q$ is a metric on $X$ with strong geometric properties. Our approach is based on the following reverse inequality for WMDMs.

Proposition 3.1. For every $x \in X$ there is $r_{x}>0$ such that

$$
q(x, y) \leqslant C_{S} \mu\left(B_{x y}\right)^{1 / 2}
$$

for all $y \in B_{d}\left(x, r_{x}\right)$, where $C_{S}=16 C_{W} C_{D}^{28+16\left\lceil\log _{2} \lambda\right\rceil}$.

Before proving Proposition 3.1, we state some consequences. We will apply the following elementary property of doubling measures, see [5, 13.1]: For all $x \in X$ and $0<r \leqslant R<\operatorname{diam}_{d}(X)$,

$$
\frac{1}{C}\left(\frac{R}{r}\right)^{1 / \alpha} \leqslant \frac{\mu\left(B_{d}(x, R)\right)}{\mu\left(B_{d}(x, r)\right)} \leqslant C\left(\frac{R}{r}\right)^{\alpha} .
$$

Here $C$ and $\alpha$ depend only on $C_{D}$.

Corollary 3.2. $(X, q)$ is a metric space homeomorphic to $(X, d)$.

Proof. Combining Proposition 3.1 and the first inequality in (2) shows that the identity map Id $:(X, d) \rightarrow(X, q)$ is locally Hölder continuous. Similarly, combining Definition 2.1 with the second inequality in (2) proves the continuity of $\mathrm{Id}^{-1}$.

We use notations $B_{d}$ and $B_{q}$ for the open balls in $(X, d)$ and $(X, q)$, respectively. We next give estimates for measures of balls in $(X, q)$.

Lemma 3.3. Let $x \in X$ and $s>0$. Then

$$
\mu\left(B_{q}(x, s)\right) \leqslant C_{W}^{2} s^{2} .
$$

Moreover, if $r_{x}>0$ is as in Proposition 3.1 and $B_{q}(x, s) \subset B_{d}\left(x, r_{x}\right)$, then

$$
\frac{s^{2}}{2 C_{S}^{2} C_{D}} \leqslant \mu\left(B_{q}(x, s)\right)
$$

Proof. First, we apply the WMDM-definition 2.1 to establish the inclusions

$$
\begin{aligned}
B_{q}(x, s) & \subset\left\{y: \mu\left(B_{x y}\right)^{1 / 2}<C_{W} s\right\} \\
& \subset\left\{y: \mu\left(B_{d}(x, d(x, y))\right)^{1 / 2}<C_{W} s\right\}=B_{d}\left(x, r_{s}\right)
\end{aligned}
$$

for some $r_{s}>0$. Since $\mu\left(B_{d}\left(x, r_{s}\right)\right) \leqslant C_{W}^{2} s^{2}$, (3) follows. Similarly, Proposition 3.1 and doubling yield

$$
\begin{aligned}
B_{q}(x, s) & \supset\left\{y: C_{S} \mu\left(B_{x y}\right)^{1 / 2}<s\right\} \\
& \supset\left\{y: C_{D}^{1 / 2} C_{S} \mu\left(B_{d}(x, d(x, y))\right)^{1 / 2}<s\right\},
\end{aligned}
$$

from which (4) follows. 
It follows from the above estimates that $\mu$ is in fact comparable to the 2-dimensional Hausdorff measure $\mathcal{H}_{q}^{2}$ in $(X, q)$. We normalize $\mathcal{H}_{q}^{2}$ so that it coincides with the Lebesgue measure if $q$ is the euclidean metric in $\mathbb{R}^{2}$.

Corollary 3.4. We have

$$
\frac{1}{2 \pi C_{S}^{2} C_{D}^{4}} \mathcal{H}_{q}^{2}(E) \leqslant \mu(E) \leqslant \frac{C_{W}^{2}}{\pi} \mathcal{H}_{q}^{2}(E)
$$

for all Borel sets $E \subset X$. In particular,

$$
\mathcal{H}_{q}^{2}\left(B_{q}(x, s)\right) \leqslant 2 \pi C_{S}^{2} C_{D}^{4} C_{W}^{2} s^{2}
$$

for all $x \in X$ and $s>0$.

Proof. The second inequality in (5) follows directly from (3) and the definition of $\mathcal{H}_{q}^{2}$. Also, (6) follows directly from (3) and the first inequality in (5).

For the first inequality in (5), we may assume that $E$ is open since $\mu$ is Radon. Given $\delta>0$, we can apply the $5 r$-covering lemma to cover $E$ with balls $B_{q}^{j}\left(x_{j}, s_{j}\right) \subset E$ satisfying (4) such that the balls $B_{q}^{j}\left(x_{j}, s_{j} / 5\right)$ are pairwise disjoint and each $s_{j}<\delta$. We denote the corresponding $\delta$-content by $\mathcal{H}_{q, \delta}^{2}$. Then by (4), the doubling property of $\mu$, and the disjointness give

$$
\begin{aligned}
\mathcal{H}_{q, \delta}^{2}(E) & \leqslant \pi \sum_{j} s_{j}^{2} \leqslant 2 \pi C_{S}^{2} C_{D} \sum_{j} \mu\left(B_{q}\left(x_{j}, s_{j}\right)\right) \\
& \leqslant 2 \pi C_{S}^{2} C_{D}^{4} \sum_{j} \mu\left(B_{q}\left(x_{j}, s_{j} / 5\right)\right) \leqslant 2 \pi C_{S}^{2} C_{D}^{4} \mu(E) .
\end{aligned}
$$

The claim follows by taking $\delta \rightarrow 0$.

\section{Proof of Proposition 3.1}

We prove Proposition 3.1 by constructing a continuum connecting the given points with controlled $q$-diameter. We define the $q$-diameter with

$$
\operatorname{diam}_{q}(A)=\sup _{a, b \in A} q(a, b)
$$

for $A \subset X$, which makes sense even though we have not yet proved that $q$ is a finite distance. Note also that the definition of $q$ implies that it satisfies the standard triangle inequality.

As a first step of the construction we find separating continua in small annuli. We denote $\ell=\left\lceil\log _{2} \lambda\right\rceil$ for the rest of this section.

Lemma 4.1. Let $x \in X$ and $r>0$ such that $\bar{B}_{d}\left(x,(2 \lambda)^{7} r\right)$ is compact and contained in a topological disk $U \subset X$. Then there exists a continuum $K \subset \bar{B}_{d}\left(x,(2 \lambda)^{6} r\right) \backslash B_{d}(x, 2 \lambda r)$ separating $B_{d}(x, r)$ and $X \backslash \bar{B}_{d}\left(x,(2 \lambda)^{7} r\right)$ with

$$
\operatorname{diam}_{q}(K) \leqslant 8 C_{W} C_{D}^{12+4 \ell} \mu\left(B_{d}(x, r)\right)^{1 / 2} .
$$


Proof. We use notation $S_{d}(x, r)=\{y \in X: d(x, y)=r\}$. Let

$$
E=S_{d}\left(x,(2 \lambda)^{3} r\right) \text { and } F=S_{d}\left(x,(2 \lambda)^{4} r\right) .
$$

By (1) and a standard compactness argument (see [7, 4.1]) there exists $\delta_{x, r}>0$ such that for all $y \in E, z \in F$ and $0<\delta<\delta_{x, r}$

$$
q^{\delta}(y, z) \geqslant \frac{1}{2 C_{W} C_{D}} \mu\left(B_{y z}\right)^{1 / 2} .
$$

Fix $0<\delta<\min \left(\delta_{x, r}, r\right)$. Using the doubling property of $\mu$ and the $5 r$-covering lemma, we can find a cover

$$
\mathcal{B}=\left\{B_{1}^{i}\right\}_{i=1}^{m}=\left\{B_{d}\left(x_{i}, r_{i}\right)\right\}_{i=1}^{m}
$$

for the annulus

$$
A=B_{d}\left(x,(2 \lambda)^{5} r\right) \backslash \bar{B}_{d}\left(x,(2 \lambda)^{2} r\right)
$$

such that the balls $B_{d}\left(x_{i}, r_{i} / 5\right)$ are pairwise disjoint, $r_{i}<\delta / 2$ and

$$
\varepsilon^{2} \leqslant \mu\left(B_{d}\left(x_{i}, r_{i} / 5\right)\right) \leqslant C_{D} \varepsilon^{2}
$$

for every $i$ and some fixed $\varepsilon>0$. Indeed, by (2) we can choose $\varepsilon$ small enough and $r_{\tilde{x}}<\delta / 2$ for every $\tilde{x} \in A$ so that (11) holds for $B_{d}\left(\tilde{x}, r_{\tilde{x}} / 5\right)$. Applying the $5 r$-covering lemma to the family of such balls yields the desired $\mathcal{B}$. The balls in the cover are contained in $B_{d}\left(x,(2 \lambda)^{6} r\right) \backslash \bar{B}_{d}(x,(2 \lambda) r)$ by the choice of $r_{i}$ and $\delta$.

If $z \in F$, there exists by the LLC-condition a continuum contained in $A$ that connects $z$ to $E$. Thus there is a chain of balls $B_{1}, \ldots, B_{n} \in \mathcal{B}$ such that for some $y \in E$ we have $y \in B_{1}, z \in B_{n}$ and $B_{j} \cap B_{j+1} \neq \emptyset$ for every $j=1, \ldots, n-1$. With this in mind, we define

$$
\mathcal{B}_{1}=\{B \in \mathcal{B}: B \cap E \neq \emptyset\}
$$

and

$$
\mathcal{B}_{j}=\left\{B \in \mathcal{B} \backslash\left(\bigcup_{k=1}^{j-1} \mathcal{B}_{k}\right): B \cap\left(\bigcup_{B^{\prime} \in \mathcal{B}_{j-1}} B^{\prime}\right) \neq \emptyset\right\} .
$$

The collections $\mathcal{B}_{j}$ form layers selected from the cover $\mathcal{B}$, the first containing those balls that intersect $E$ and the subsequent ones those not previously selected which intersect with the previous layer.

Recall that each $z \in F$ is contained in some $B_{z} \in \mathcal{B}_{n}$, where $n$ depends on $z$. We claim that

$$
n \geqslant \frac{\sqrt{m}}{4 C_{W} C_{D}^{6+\ell}}
$$

for all such $z$, where $m$ is the number of balls in the cover (10). Indeed, if $B_{1}, \ldots, B_{n}$ is a chain of balls as above, then their centers and the points $y$ and $z$ form a $\delta$-chain. Thus, if we denote $B_{0}=B\left(y, r\left(B_{1}\right)\right)$ and 
$B_{n+1}=B\left(z, r\left(B_{n}\right)\right)$, then (9), the definition of $q^{\delta}(y, z)$, the doubling property of $\mu$, and (11) yield

$$
\begin{aligned}
\mu\left(B_{y z}\right)^{1 / 2} & \leqslant 2 C_{W} C_{D} q^{\delta}(y, z) \leqslant 2 C_{W} C_{D} \sum_{j=0}^{n+1} \mu\left(B_{j}\right)^{1 / 2} \\
& \leqslant 4 C_{W} C_{D}^{2} \sum_{j=1}^{n} \mu\left(B_{j}\right)^{1 / 2} \leqslant 4 C_{W} C_{D}^{4} n \varepsilon .
\end{aligned}
$$

But since $\lambda \geqslant 1$, we have $d(y, z) \geqslant(2 \lambda)^{4} r-(2 \lambda)^{3} r \geqslant 2^{3} \lambda^{4} r$ by our choices of these points and $E, F$ in (8). Therefore, the triangle inequality yields

$$
B_{d}\left(x,(2 \lambda)^{6} r\right) \subset B_{d}\left(y, 2(2 \lambda)^{6} r\right) \subset B_{d}\left(y, 4(2 \lambda)^{2} d(y, z)\right) .
$$

As the $m$ balls $B_{d}\left(x_{i}, r_{i} / 5\right)$ in $\mathcal{B}$ are pairwise disjoint, combining the doubling property of $\mu$ with (10), (11) and (13) yields

$$
\mu\left(B_{y z}\right) \geqslant \frac{\mu\left(B_{d}\left(y, 4(2 \lambda)^{2} d(y, z)\right)\right)}{C_{D}^{4+2 \ell}} \geqslant \frac{\mu\left(B_{d}\left(x,(2 \lambda)^{6} r\right)\right)}{C_{D}^{4+2 \ell}} \geqslant \frac{m \varepsilon^{2}}{C_{D}^{4+2 \ell}},
$$

(recall that $\ell=\left\lceil\log _{2} \lambda\right\rceil$ ) so (12) follows.

Let $n_{0}=\left\lceil\sqrt{m} / 4 C_{W} C_{D}^{6+\ell}\right\rceil$. As the layers $\mathcal{B}_{j}$ are pairwise disjoint, we have

$$
\begin{aligned}
\sum_{j=1}^{n_{0}} \sum_{B_{i} \in \mathcal{B}_{j}} \mu\left(B_{i}\right)^{1 / 2} & \leqslant C_{D}^{2} m \varepsilon \leqslant 4 C_{W} C_{D}^{8+\ell} n_{0} \sqrt{m} \varepsilon \\
& \leqslant 4 C_{W} C_{D}^{11+4 \ell} n_{0} \mu\left(B_{d}(x, r)\right)^{1 / 2} .
\end{aligned}
$$

Hence for some $1 \leqslant j_{0} \leqslant n_{0}$ we have

$$
\sum_{B_{i} \in \mathcal{B}_{j_{0}}} \mu\left(B_{i}\right)^{1 / 2} \leqslant C^{\prime} \mu\left(B_{d}(x, r)\right)^{1 / 2}
$$

where $C^{\prime}=4 C_{W} C_{D}^{11+4 \ell}$. We denote by $K_{1}^{\prime}$ the compact set $\cup_{B_{i} \in \mathcal{B}_{j_{0}}} \bar{B}_{i}$. By the choice of $n_{0}$ and the LLC-condition $K_{1}^{\prime}$ separates $B_{d}(x, r)$ and $X \backslash \bar{B}_{d}\left(x,(2 \lambda)^{7} r\right)$. Moreover, since $\bar{B}_{d}\left(x,(2 \lambda)^{7} r\right) \subset U$ for some $U \subset X$ homeomorphic to a disk, a component $K_{1}$ of $K_{1}^{\prime}$ also separates the same sets (see for example [9, V 14.3]).

By repeating the above construction for $\delta / j, j=2,3, \ldots$ we obtain continua $K_{j}$, each separating $B_{d}(x, r)$ and $X \backslash \bar{B}_{d}\left(x,(2 \lambda)^{7} r\right)$. By connectedness, between any two points of $K_{j}$ there exists a $\delta / j$-chain among the centers of the balls $B_{j}^{i}$ covering $K_{j}$. For each $j$ we have the same estimate

$$
\sum_{i} \mu\left(B_{j}^{i}\right)^{1 / 2} \leqslant C^{\prime} \mu\left(B_{d}(x, r)\right)^{1 / 2}
$$

Then using compactness in the Hausdorff metric for compact sets we find a subsequence of $\left(K_{j}\right)$ converging to a compact set $K^{\prime}$. Now also 
$K^{\prime}$ and hence one of its components $K$ again separates $B_{d}(x, r)$ and $X \backslash \bar{B}_{d}\left(x,(2 \lambda)^{7} r\right)$.

If $a, b \in K$ and $\delta^{\prime}>0$, we pick a large $j$ such that $\delta / j<\delta^{\prime}$ and the Hausdorff distance between $K$ and $K_{j}$ is less than $\delta^{\prime}$. Then from $K_{j}$ we find points $p_{1}, \ldots, p_{l-1}$ so that $a$ and $b$ are connected by the $\delta^{\prime}$-chain $a=p_{0}, p_{1}, \ldots, p_{l-1}, p_{l}=b$ with

$$
q^{\delta^{\prime}}(a, b) \leqslant \sum_{i=1}^{l} \mu\left(B_{p_{i} p_{i-1}}\right)^{1 / 2} \leqslant 2 C_{D} C^{\prime} \mu\left(B_{d}(x, r)\right)^{1 / 2} .
$$

Since the upper bound holds for all $\delta^{\prime}>0$ the estimate is true also for $q(a, b)$.

For $x \in X, r>0$ and $K$ as in Lemma 4.1, we let

$$
\begin{aligned}
& K(x, r)=K \text { and } \\
& \hat{K}(x, r)=\text { the component of } X \backslash K(x, r) \text { containing } x .
\end{aligned}
$$

The following lemma on basic planar topology allows us to connect $K\left(x_{1}, r_{1}\right)$ and $K\left(x_{2}, r_{2}\right)$ for correctly chosen adjacent balls $B_{d}\left(x_{1}, r_{1}\right)$ and $B_{d}\left(x_{2}, r_{2}\right)$. We refer to $[7,5.1]$ for a proof.

Lemma 4.2. Let $x_{1}, x_{2} \in X$ and $r_{1}, r_{2}>0$ be as in Lemma 4.1 such that

(1) $\hat{K}\left(x_{1}, r_{1}\right)$ and $\hat{K}\left(x_{2}, r_{2}\right)$ intersect,

(2) $\hat{K}\left(x_{1}, r_{1}\right) \not \subset \hat{K}\left(x_{2}, r_{2}\right)$,

(3) $\hat{K}\left(x_{2}, r_{2}\right) \not \subset \hat{K}\left(x_{1}, r_{1}\right)$.

Then the continua $K\left(x_{1}, r_{1}\right)$ and $K\left(x_{2}, r_{2}\right)$ intersect.

With these lemmas we are ready to construct the desired continuum between the given points.

Proof of Proposition 3.1. Let $y \in X$ be such that $B_{d}(x, 2 \lambda d(x, y))$ is contained in a topological disk. Then, as in the proof of Lemma 4.1, we can cover the ball $B_{1}=B_{d}(x, \lambda d(x, y))$ with $M_{1}$ balls

$$
B_{1}^{i}=B_{d}\left(z_{i}^{1}, r_{i}^{1}\right)
$$

such that $z_{i}^{1} \in B_{1}$, the balls $\frac{1}{5} B_{1}^{i}$ are pairwise disjoint, and

$$
\mu\left(B_{1}\right) / 4 C_{D}^{8+7 \ell} \leqslant \mu\left((2 \lambda)^{7} B_{1}^{i}\right) \leqslant \mu\left(B_{1}\right) / 4 C_{D}^{7+7 \ell}
$$

for each $i$. The doubling condition and (14) now imply that

$$
M_{1} \leqslant C_{D}^{19+14 \ell}
$$

and that

$$
(2 \lambda)^{7} r_{i}^{1} \leqslant \frac{\lambda \operatorname{radius}\left(B_{1}\right)}{4}
$$

Furthermore, Lemma 4.1 can be applied with $z_{i}^{1}$ and $r_{i}^{1}$ for each $i$.

Let $\mathcal{I}_{1}$ be the set of indices $i$ such that $B_{1}^{i}$ intersects the component $D_{1}$ of $B_{1}$ containing $x$ and $\hat{K}\left(z_{i}^{1}, r_{i}^{1}\right) \not \subset \hat{K}\left(z_{j}^{1}, r_{j}^{1}\right)$ for all $j \neq i$. For 
future reference, notice that $y \in D_{1}$ by the LLC-condition. Then the compact set

$$
K_{1}=\bigcup_{i \in \mathcal{I}_{1}} K\left(z_{i}^{1}, r_{i}^{1}\right) \subset 2 B_{1}
$$

is connected. Indeed, if $k, l \in \mathcal{I}_{1}$, there exists a path from $\hat{K}\left(z_{k}^{1}, r_{k}^{1}\right)$ to $\hat{K}\left(z_{l}^{1}, r_{l}^{1}\right)$ in $B_{1}$. This path is now covered by a chain of sets $\hat{K}\left(z_{i}^{1}, r_{i}^{1}\right)$, $i \in \mathcal{I}_{1}$, so that for consecutive members in the chain the corresponding continua $K\left(z_{i}^{1}, r_{i}^{1}\right)$ intersect by Lemma 4.2 and the choice of $\mathcal{I}_{1}$.

Next we choose $h \in \mathcal{I}_{1}$ such that $x \in \hat{K}\left(z_{h}^{1}, r_{h}^{1}\right)$ and denote

$$
B_{2}=B_{d}\left(z_{h}^{1},(2 \lambda)^{7} r_{h}^{1}\right) \text {. }
$$

Then $x \in B_{2}$ and $2 B_{2} \subset \frac{1}{2} B_{1}$. We cover $B_{2}$ with $M_{2}$ balls

$$
B_{2}^{i}=B_{d}\left(z_{i}^{2}, r_{i}^{2}\right)
$$

so that all the properties above remain valid with the balls $B_{1}^{i}$ replaced by the balls $B_{2}^{i}$. In particular, (14) takes the form

$$
\mu\left(B_{2}\right) / 4 C_{D}^{8+7 \ell} \leqslant \mu\left((2 \lambda)^{7} B_{2}^{i}\right) \leqslant \mu\left(B_{2}\right) / 4 C_{D}^{7+7 \ell} .
$$

Repeating the previous construction then yields continuum

$$
K_{2}=\bigcup_{i \in \mathcal{I}_{2}} K\left(z_{i}^{2}, r_{i}^{2}\right) \subset 2 B_{2} .
$$

We next show that

$$
K_{1} \cap K_{2} \neq \emptyset .
$$

First, if $K\left(z_{i}^{2}, r_{i}^{2}\right)$ is one of the continua in (16), then $\hat{K}\left(z_{h}^{1}, r_{h}^{1}\right) \not \subset$ $\hat{K}\left(z_{i}^{2}, r_{i}^{2}\right)$, since otherwise we would have

$$
B_{d}\left(z_{h}^{1}, r_{h}^{1}\right) \subset B_{d}\left(z_{i}^{2},(2 \lambda)^{7} r_{i}^{2}\right)
$$

and by (14)

$$
\mu\left(B_{d}\left(z_{h}^{1}, r_{h}^{1}\right)\right) \leqslant \mu\left(B_{2}\right) / 4 C_{D}^{7+7 \ell} \leqslant \mu\left(B_{d}\left(z_{h}^{1}, r_{h}^{1}\right)\right) / 4,
$$

a contradiction.

Secondly, if

$$
w \in \overline{\hat{K}\left(z_{h}^{1}, r_{h}^{1}\right)} \cap K\left(z_{h}^{1}, r_{h}^{1}\right)
$$

then at least one of the sets $K\left(z_{i}^{2}, r_{i}^{2}\right)$ in (16) satisfies $w \in \hat{K}\left(z_{i}^{2}, r_{i}^{2}\right)$. Then also

$$
\hat{K}\left(z_{i}^{2}, r_{i}^{2}\right) \not \subset \hat{K}\left(z_{h}^{1}, r_{h}^{1}\right) \quad \text { and } \quad \hat{K}\left(z_{h}^{1}, r_{h}^{1}\right) \cap \hat{K}\left(z_{i}^{2}, r_{i}^{2}\right) \neq \emptyset .
$$

Thus

$$
K\left(z_{h}^{1}, r_{h}^{1}\right) \cap K\left(z_{i}^{2}, r_{i}^{2}\right) \neq \emptyset
$$

by Lemma 4.2, and (17) follows.

We continue the above process to obtain continua

$$
K_{j}=\bigcup_{i \in \mathcal{I}_{j}} K\left(z_{i}^{j}, r_{i}^{j}\right) \subset 2 B_{j}
$$


for each $j \in \mathbb{N}$, such that $K_{j} \subset 2 B_{j} \ni x$ for all $j$, and

$$
\operatorname{diam}_{d}\left(B_{j}\right) \rightarrow 0 \text { as } j \rightarrow \infty .
$$

Moreover, applying the constructions of the balls $B_{j}$ together with estimates (14) applied to these balls, we get

$$
\mu\left(B_{j+1}\right)^{1 / 2} \leqslant \frac{1}{2} \mu\left(B_{j}\right)^{1 / 2}
$$

Repeating the argument in the previous paragraphs, we see that $K_{j} \cap$ $K_{j+1} \neq \emptyset$ for all $j$. Therefore,

$$
K=\cup_{j=1}^{\infty} K_{j} \cup\{x\}
$$

is a continuum.

We now apply (7) and the construction of the set $K$ to estimate its $q$-diameter. First, if $a, b \in K_{1}$, then for some $i_{1}, \ldots, i_{m+1} \in \mathcal{I}_{1}$ and points $x_{1}, \ldots, x_{m} \in K_{1}$ we have $a \in K\left(z_{i_{1}}^{1}, r_{i_{1}}^{1}\right)$,

$x_{1} \in K\left(z_{i_{1}}^{1}, r_{i_{1}}^{1}\right) \cap K\left(z_{i_{2}}^{1}, r_{i_{2}}^{1}\right), \ldots, x_{m} \in K\left(z_{i_{m}}^{1}, r_{i_{m}}^{1}\right) \cap K\left(z_{i_{m+1}}^{1}, r_{i_{m+1}}^{1}\right)$, and $b \in K\left(z_{i_{m+1}}^{1}, r_{i_{m+1}}^{1}\right)$. By $(7)$,

$$
\begin{aligned}
q(a, b) & \leqslant q\left(a, x_{1}\right)+q\left(b, x_{m}\right)+\sum_{j=1}^{m-1} q\left(x_{j}, x_{j+1}\right) \\
& \leqslant 8 C_{W} C_{D}^{12+4 \ell} \sum_{j=1}^{m+1} \mu\left(B\left(z_{i_{j}}^{1}, r_{i_{j}}^{1}\right)\right)^{1 / 2} .
\end{aligned}
$$

Since $m+1 \leqslant M_{1}$, combining with (14) and (15) gives

$$
q(a, b) \leqslant C_{2} \mu\left(B_{1}\right)^{1 / 2},
$$

where $C_{2}=4 C_{W} C_{D}^{28+15 \ell}$. In particular, we get an upper bound for the $q$-diameter of $K_{1}$. Repeating the argument, we get

$$
\operatorname{diam}_{q}\left(K_{j}\right) \leqslant C_{2} \mu\left(B_{j}\right)^{1 / 2}
$$

for all $j$. Combining with (18), we moreover have

$$
\operatorname{diam}_{q}\left(K_{j}\right) \leqslant 2^{1-j} C_{2} \mu\left(B_{1}\right)^{1 / 2}
$$

for each $j$.

Now let $w_{0} \in K_{1}$. Fix $\delta>0, \varepsilon>0$, and

$$
w_{j} \in K_{j} \cap K_{j+1}
$$

for each $j \geqslant 1$. Since $d\left(w_{j}, x\right) \rightarrow 0$, we find $k \in \mathbb{N}$ such that $d\left(w_{k}, x\right)<\delta$ and $\mu\left(B_{w_{k} x}\right)^{1 / 2}<\varepsilon$. Then, by (19),

$$
q^{\delta}\left(w_{0}, x\right) \leqslant \sum_{j=1}^{k} \operatorname{diam}_{q}\left(K_{j}\right)+q^{\delta}\left(w_{k}, x\right) \leqslant 2 C_{2} \mu\left(B_{1}\right)^{1 / 2}+\varepsilon
$$

and hence

$$
q\left(w_{0}, x\right) \leqslant 2 C_{2} \mu\left(B_{1}\right)^{1 / 2}
$$


Finally, recall that our goal is to bound $q(x, y)$. Since $y \in D_{1}$, we can repeat the argument above with the same cover for $B_{1}$ to find that (20) holds with $x$ replaced by $y$. By triangle inequality, we conclude that

$$
q(x, y) \leqslant 4 C_{2} \mu\left(B_{1}\right)^{1 / 2} \leqslant 4 C_{D}^{\ell} C_{2} \mu\left(B_{x y}\right)^{1 / 2} .
$$

The proof is complete.

\section{QUASICONFORMAL UNIFORMIZATION}

Our strategy for proving Theorem 2.2 is to apply the existence of a quasiconformal homeomorphism $f$ from $(X, q)$ to a circle domain $\Omega$. This is guaranteed by a recent result of Ikonen [6] and the classical Koebe uniformization of finitely connected Riemann surfaces. We will show in Sections 6 and 7 that $f$ is in fact quasisymmetric, with respect to the original metric $d$, under a suitable normalization.

We recall the geometric definition of quasiconformal maps. Let $Y=$ $(Y, d)$ be a metric space such that $\mathcal{H}_{d}^{2}$ is finite on compact subsets. We moreover assume that $Y$ is a topological 2-manifold. It then follows that $\mathcal{H}_{d}^{2}$ is positive on open sets, cf. [10].

Let $\Gamma$ be a family of paths in $Y$. We say that a Borel function $\rho \geqslant 0$ in $Y$ is admissible for $\Gamma$, if

$$
\int_{\gamma} \rho d s \geqslant 1 \text { for all locally rectifiable } \gamma \in \Gamma .
$$

The (conformal) modulus of $\Gamma$ is

$$
\bmod (\Gamma)=\inf \int_{Y} \rho^{2} d \mathcal{H}_{d}^{2}
$$

where the infimum is taken over all admissible functions.

A homeomorphism $f: Y \rightarrow Z$ between spaces as above is (geometric) $K$-quasiconformal, $K \geqslant 1$, if

$$
K^{-1} \bmod (\Gamma) \leqslant \bmod (f \Gamma) \leqslant K \bmod (\Gamma)
$$

for all path families $\Gamma$ in $Y$, where $f \Gamma=\{f \circ \gamma: \gamma \in \Gamma\}$.

It is shown in [10] and [11] that if $Y$ is a topological disk for which there exists $C>0$ such that

$$
\mathcal{H}_{d}^{2}\left(B_{d}(y, r)\right) \leqslant C r^{2} \quad \text { for all } y \in Y, r>0,
$$

then there exists a $\pi / 2$-quasiconformal homeomorphism from $Y$ into the euclidean plane. Recently Ikonen [6] generalized this result to the case of non-simply connected surfaces. In particular, he showed that the upper bound (6) guarantees that there is a $\pi / 2$-quasiconformal homeomorphism from our space $(X, q)$ onto a Riemann surface $Z$. Moreover, by the classical uniformization theorem for finitely connected Riemann surfaces, there is a conformal map from $Z$ onto a circle domain $\Omega$. Recall that conformal maps are 1-quasiconformal in the sense of the geometric definition above, and that the composition of a $K_{1^{-}}$ 
and a $K_{2}$-quasiconformal map is $K_{1} K_{2}$-quasiconformal. Thus we have the following.

Proposition 5.1. There is a $\pi / 2$-quasiconformal homeomorphism $f$ : $(X, q) \rightarrow \Omega$, where $\Omega \subset \mathbb{S}^{2}$ is a circle domain. If moreover $(X, q)$ is not homeomorphic to $\mathbb{S}^{2}$, then the statement remains valid with circle domain $\Omega \subset \mathbb{R}^{2}$.

The second statement follows from the first simply by postcomposing $f$ with a suitable Möbius transformation followed with the stereographic projection.

\section{Modulus estimate in CIRCle Domains}

In this section we assume that $\bar{X} \backslash X$ has at least two components. Let $\Omega \subset \mathbb{R}^{2}$ be the circle domain in Proposition 5.1. We prove a Loewner-type modulus estimate, Proposition 6.1, which along with Proposition 3.1 is the main technical result towards Theorem 2.2.

Proposition 6.1 is related to the work of Bonk [1] on the uniformization of Sierpiński carpets in the plane. There a Loewner estimate is proved for domains whose boundary components are quasicircles that are suitably separated ([1, Proposition 7.5]). The estimate in Proposition 6.1, which is also in terms of the separation of the boundary components, is more precise but only holds in the more restrictive setting of circle domains.

In what follows, we denote by $\Gamma(A, B ; G)$ the family of paths joining sets $A, B \subset \bar{G}$ in $G$, i.e., all the paths $\gamma:[a, b] \rightarrow \bar{G}$ such that $\gamma(a) \in A, \gamma(b) \in B$, and $\gamma(t) \in G$ for all $a<t<b$. We abbreviate $\bmod (A, B ; G)=\bmod (\Gamma(A, B ; G))$.

Proposition 6.1. Let $E_{1}, E_{2} \subset \Omega$ be disjoint continua such that

$$
\frac{\min \left\{\operatorname{diam}\left(E_{1}\right), \operatorname{diam}\left(E_{2}\right)\right\}}{\operatorname{dist}\left(E_{1}, E_{2}\right)} \geqslant 1 \text {. }
$$

Then

$$
\bmod \left(E_{1}, E_{2} ; \Omega\right) \geqslant \frac{\alpha^{M}}{2 \pi(10 M)^{M}(M+2)^{2}}
$$

where

$$
\alpha=2^{-2-2 M-\pi^{2} C_{W}^{2} C_{D}^{1+\log _{2} C_{X}} / 8 \log 2} .
$$

The rest of this section is devoted to the proof of Proposition 6.1. We denote the complementary components of $\Omega$ by

$$
D_{1}, \ldots, D_{M}, \quad D_{i}=\bar{D}\left(z_{i}, r_{i}\right) .
$$

Complementary point-components do not have effect on the modulus. Therefore, we can assume that $r_{i}>0$ for all $i$. We use notation

$$
\Delta(i, j)=\frac{\operatorname{dist}\left(D_{i}, D_{j}\right)}{\min \left\{r_{i}, r_{j}\right\}}
$$


for the relative distances. The homeomorphism $f$ in Proposition 5.1 uniquely extends to a bijection from the set of components of $\bar{X} \backslash X$ to the set $\left\{D_{i}\right\}$. We denote by $A_{i}$ the component corresponding to $D_{i}$ under this bijection.

Lemma 6.2. We have $\Delta(i, j) \geqslant \alpha$ for every $i \neq j$, where $\alpha$ is the constant in Proposition 6.1.

Proof. Fix $i \neq j$ such that $r_{i} \leqslant r_{j}$. We consider $\bmod \left(D_{i}, D_{j} ; \Omega\right)$. We first claim that

$$
\bmod \left(D_{i}, D_{j} ; \Omega\right) \leqslant \frac{\pi}{2} \bmod \left(A_{i}, A_{j} ; X\right) \leqslant \frac{\pi C_{W}^{2} C_{D}^{1+\log _{2} C_{X}}}{2} .
$$

The first inequality follows from the quasiconformality of $f$. Towards the second inequality, recall that $C_{X}$ is the ratio of the diameter of $(X, d)$ to the minimum $d$-distance $D$ between the components $A_{i}$. Let $m \geqslant 1$ be the smallest integer such that $C_{X} \leqslant 2^{m}$. Then, by the WMDM-condition and the doubling property of $\mu$, the length of every path in $\Gamma\left(A_{i}, A_{j} ; X\right)$ is at least

$$
C_{W}^{-1} \inf _{x \in X} \mu(B(x, D))^{1 / 2} \geqslant C_{W}^{-1} C_{D}^{-m / 2} \mu(X)^{1 / 2} .
$$

Therefore,

$$
\bmod \left(A_{i}, A_{j} ; X\right) \leqslant \int_{X} C_{W}^{2} C_{D}^{m} \mu(X)^{-1} d \mu=C_{W}^{2} C_{D}^{m},
$$

and (23) follows. We prove the lower bound for $\Delta(i, j)$ by showing that the opposite of $(23)$ holds if $\Delta(i, j)<\alpha$.

Let $s=\operatorname{dist}\left(D_{i}, D_{j}\right)$ and

$$
w=z_{i}+\frac{\left(r_{i}+\frac{s}{2}\right)\left(z_{j}-z_{i}\right)}{\left|z_{j}-z_{i}\right|}
$$

be the point in the middle of $D_{i}$ and $D_{j}$. If $\Delta(i, j)<\alpha$, we have

$$
2^{N+2} s \leqslant r_{i},
$$

where $N=\left\lfloor 2 M+\pi^{2} C_{W}^{2} C_{D}^{1+\log _{2} C_{X}} / 8 \log 2\right\rfloor$. We consider the path families

$$
\Psi_{n}=\left\{\text { components of } S(w, t s) \cap \bar{\Omega}: 2^{n-1}<t<2^{n}\right\}
$$

for $n=1, \ldots, N$. Every path in $\Psi_{0} \cup \cdots \cup \Psi_{N}$ either connects $D_{i}$ and $D_{j}$ or intersects some $D_{k}, k \neq i, j$. We claim that any such $D_{k}=\bar{D}\left(z_{k}, r_{k}\right)$ intersects paths from at most two families $\Psi_{n}$.

Suppose to the contrary that $D_{k}$ intersects paths from $\Psi_{n}$ and $\Psi_{n+2}$ for some $n$. Then there exist $w_{1}, w_{2} \in D_{k}$ with

$$
\left|w_{1}-w\right|<2^{n} s \text { and }\left|w_{2}-w\right|>2^{n+1} s
$$

so that

$$
2^{n-1} s \leqslant r_{k} .
$$


Using basic planar geometry, (24) and (25), we have

$$
\left|z_{k}-z_{i}\right|^{2} \leqslant\left(r_{i}+\frac{s}{2}\right)^{2}+\left(r_{k}+2^{n-1} s\right)^{2}<\left(r_{i}+r_{k}\right)^{2} .
$$

But this is impossible since $D_{i}$ and $D_{k}$ are disjoint.

Since the number of disks $D_{k}, k \neq i, j$ is at most $M$, we have shown that for at least $N-2 M+2$ different indices $n$ all the paths in $\Psi_{n}$ connect $D_{i}$ and $D_{j}$. Using standard properties of the modulus we have then the lower bounds

$$
\bmod \left(\Psi_{n}\right) \geqslant 4 \bmod (\{S(0, t): 1<t<2\}) \geqslant \frac{2 \log 2}{\pi}
$$

for every $n$, see [12, Theorem 10.12], and

$$
\bmod \left(D_{i}, D_{j} ; \Omega\right) \geqslant(N-2 M+2) \frac{2 \log 2}{\pi}>\frac{\pi C_{W}^{2} C_{D}^{1+\log _{2} C_{X}}}{4} .
$$

We have thus proved that $\Delta(i, j)<\alpha$ leads to a contradiction with (23), and the lemma follows.

Recall that $D_{i}=\bar{D}\left(z_{i}, r_{i}\right)$. We next consider the sets

$$
\Phi_{i}=\left\{1<t<1+\alpha: S\left(z_{i}, t r_{i}\right) \subset \Omega\right\},
$$

where $\alpha$ is the constant in Proposition 6.1, and the family $\Gamma_{i}$ of all the (parameterized) circles $S\left(z_{i}, t r_{i}\right), t \in \Phi_{i}$.

Lemma 6.3. We have

$$
m_{1}\left(\Phi_{i}\right) \geqslant \frac{\alpha^{M}}{(10 M)^{M}}
$$

for all $i=1, \ldots, M$. In particular,

$$
\bmod \left(\Gamma_{i}\right) \geqslant \beta=\frac{\alpha^{M}}{2 \pi(10 M)^{M}} .
$$

Proof. Enumerate the disks according to decreasing radius, and fix $D_{i}$. By Lemma $6.2, \operatorname{dist}\left(D_{i}, D_{j}\right) \geqslant \alpha r_{i}$ for every $j<i$. Now, if

$$
\sum_{j \geqslant i+1} r_{j} \leqslant \alpha r_{i} / 10
$$

then (27) holds. Otherwise $r_{i+1} \geqslant \alpha r_{i} /(10 M)$. Continuing inductively, either

$$
\sum_{j \geqslant i+k+1} r_{j} \leqslant \alpha r_{i+k} / 10
$$

for some $k$, or

$$
r_{i+k+1} \geqslant \alpha r_{i+k} /(10 M) \geqslant \ldots \geqslant r_{i} \alpha^{k+1} /(10 M)^{k+1}
$$

for all $k$. In the latter case,

$$
r_{j} \geqslant r_{i} \alpha^{M-1} /(10 M)^{M-1} \text { for all } j=1, \ldots, M,
$$


and (27) follows from Lemma 6.2. On the other hand, if (29) occurs then Lemma 6.2 shows that

$$
r_{i} m_{1}\left(\Phi_{i}\right) \geqslant \min _{j \leqslant i+k} \operatorname{dist}\left(D_{i}, D_{j}\right)-\sum_{j \geqslant i+k+1} 2 r_{j} \geqslant \frac{\alpha r_{i+k}}{10} .
$$

If moreover $k$ is the smallest index for which (29) occurs, then (30) holds for $j$ replaced with $i+k$ and we conclude (27) also in this case. Finally, (28) follows from (27) by a standard application of Hölder's inequality and polar coordinates.

Now fix continua $E_{1}, E_{2}$ as in Proposition 6.1. First, an elementary geometric argument (cf. [12, Theorem 11.7]) applying (21) shows that there exist $z_{0} \in \mathbb{R}^{2}$ and $r_{0}>0$ such that both $E_{1}$ and $E_{2}$ intersect $S\left(z_{0}, t_{0} r_{0}\right)$ for all $1 \leqslant t_{0} \leqslant \sqrt{3}$.

Let $\Phi_{i}, \Gamma_{i}, i=1, \ldots, M$, be as in Lemma 6.3. Moreover, we denote $\Phi_{0}=(1, \sqrt{3})$ and

$$
\Gamma_{0}=\left\{S\left(z_{0}, t_{0} r_{0}\right): t_{0} \in \Phi_{0}\right\},
$$

so that (28) holds for all $i=0, \ldots, M$. By construction, $\Gamma_{i}$ is a family of paths in $\Omega$ when $i=1, \ldots, M$, while the paths in $\Gamma_{0}$ are not required to lie in $\Omega$. The proposition is proved by modifying $\Gamma_{0}$ to obtain a family of paths in $\Omega$ such that the lower bound for modulus is still valid. This is based on the following property.

Lemma 6.4. Given

$$
T=\left(t_{0}, t_{1}, \ldots, t_{M}\right) \in \Phi:=\Phi_{0} \times \Phi_{1} \times \cdots \times \Phi_{M},
$$

there is an injective path $\gamma_{T}$ connecting $E_{1}$ and $E_{2}$ in

$$
G_{T}:=\bigcup_{j=0}^{M} S\left(z_{j}, t_{j} r_{j}\right) \cap \Omega .
$$

Proof. By construction, there are $p_{1}, p_{2} \in S\left(z_{0}, t_{0} r_{0}\right)$ such that $p_{1} \in E_{1}$ and $p_{2} \in E_{2}$. On the other hand, the components of $S\left(z_{0}, t_{0} r_{0}\right) \backslash \Omega$ are of the form

$$
S\left(z_{0}, t_{0} r_{0}\right) \cap D_{j}=S\left(z_{0}, t_{0} r_{0}\right) \cap \bar{D}\left(z_{j}, r_{j}\right) .
$$

Therefore, the $p_{1}$-component $F_{T}$ of $G_{T}$ contains all of $S\left(z_{0}, t_{0} r_{0}\right) \cap \Omega$. In particular, it contains $p_{2}$. We can choose $\gamma_{T}$ to be a shortest path joining $p_{1}$ and $p_{2}$ in $F_{T}$.

Let

$$
\Gamma=\left\{\gamma_{T}: T \in \Phi\right\},
$$

where $\gamma_{T}$ is any path satisfying the conditions of Lemma 6.4. The proposition follows if we can bound $\bmod (\Gamma)$ from below.

Let $\rho \geqslant 0$ be a Borel function in $\Omega$ such that

$$
\int_{\Omega} \rho^{2} d A=\frac{\beta}{(M+2)^{2}},
$$


where $\beta$ is the constant in (28). The desired lower bound follows if we can show that such a $\rho$ cannot be admissible for $\Gamma$. By (28), (31), and (32), we find that $(M+3 / 2) \rho$ cannot be admissible for any of the path families $\Gamma_{i}, i=0, \ldots, M$. Hence there is at least one $T=$ $\left(t_{0}, t_{1}, \ldots, t_{M}\right) \in \Phi$ such that

$$
\int_{S\left(z_{i}, t_{i} r_{i}\right)} \rho d s<\frac{1}{M+3 / 2}
$$

for each $i=0, \ldots, M$. Applying the injectivity of $\gamma_{T}$, we moreover get

$$
\int_{\gamma_{T}} \rho d s \leqslant \sum_{i=0}^{M} \int_{S\left(z_{i}, t_{i} r_{i}\right)} \rho d s \leqslant \frac{M+1}{M+3 / 2}<1 .
$$

We conclude that $\rho$ is not admissible for $\Gamma$. The proof of Proposition 6.1 is complete.

\section{Proof of Theorem 2.2}

Suppose that the assumptions of Theorem 2.2 are valid. By Proposition 5.1, there is a $\pi / 2$-quasiconformal map $f:(X, q) \rightarrow \Omega$, where $\Omega \subset \mathbb{S}^{2}$ is a circle domain. We prove Theorem 2.2 by showing that, after a normalization, $f$ is quasisymmetric with respect to the original metric $d$.

If $\Omega=\mathbb{S}^{2}$, then the theorem is proved in [7]. The proof in the case of one complementary component is easier than the one below and is omitted. We consider the remaining case where there are at least two complementary components.

Recall that the assumptions of Väisälä's theorem ([5, Theorem 10.17]) hold in our setting, so the quasisymmetry of $f$ follows if we can prove the weak quasisymmetry of $h=f^{-1}$ : there is $t \geqslant 1$ such that for every disjoint $y_{0}, y_{1}, y_{2} \in \Omega$ with

$$
\left|y_{0}-y_{1}\right| \leqslant\left|y_{0}-y_{2}\right| \leqslant \frac{1}{10}
$$

we have

$$
d\left(h\left(y_{0}\right), h\left(y_{1}\right)\right) \leqslant t d\left(h\left(y_{0}\right), h\left(y_{2}\right)\right) .
$$

To prove (33), we first normalize $h$. Namely, we precompose $h$ with a suitable Möbius transformation, if necessary, so that

$$
\min _{i \neq j} d\left(h\left(a_{i}\right), h\left(a_{j}\right)\right) \geqslant \operatorname{diam}_{d}(X) / 10,
$$

where $\left\{a_{0}, a_{1}, a_{\infty}\right\} \in \Omega$ correspond to the points $0, e_{1}$, and $\infty$ under the stereographic projection.

Fix $y_{0}, y_{1}, y_{2} \in \Omega$ as in (33), and denote

$$
A=d\left(h\left(y_{0}\right), h\left(y_{2}\right)\right), \quad B=d\left(h\left(y_{0}\right), h\left(y_{1}\right)\right) .
$$

We need to show that $B \leqslant t A$. We may assume that

$$
A \leqslant B / 100 \lambda^{3} \leqslant \operatorname{diam}_{d}(X) / 100 \lambda^{3},
$$


otherwise there is nothing to prove. By (34) and triangle inequality, we find that for some $j \in\{0,1, \infty\}$,

$$
d\left(h\left(y_{0}\right), h\left(a_{j}\right)\right) \geqslant \operatorname{diam}_{d}(X) / 20 \text { and }\left|y_{1}-a_{j}\right| \geqslant 1 / 10 .
$$

Moreover, by the LLC-condition, we find a continuum

$$
F_{1} \subset B_{d}\left(h\left(y_{0}\right), \lambda A\right) \subset X
$$

joining $h\left(y_{0}\right)$ and $h\left(y_{2}\right)$. Similarly, we find a continuum

$$
F_{2} \subset X \backslash B_{d}\left(h\left(y_{0}\right), B / \lambda\right)
$$

joining $h\left(y_{1}\right)$ and $h\left(a_{j}\right)$.

Denote $E_{\ell}=f\left(F_{\ell}\right)=h^{-1}\left(F_{\ell}\right)$ for $\ell=1,2$. Then, if $\tau$ is a rotation of $\mathbb{S}^{2}$ sending $y_{0}$ to 0 and $\phi$ the stereographic projection, we see that $(\phi \circ$ $\tau)\left(E_{1}\right)$ and $(\phi \circ \tau)\left(E_{2}\right)$ satisfy the conditions of Proposition 6.1. Since $\phi \circ \tau$ is conformal, we conclude that the lower bound in Proposition 6.1 holds also for the continua $E_{1}$ and $E_{2}$.

Next, we estimate $\bmod \left(F_{1}, F_{2} ; X\right)$ from above (recall the definition from Section 5). Denote

$$
U_{1}=\bar{B}_{d}\left(h\left(y_{0}\right), \lambda A\right), \quad U_{2}=X \backslash B_{d}\left(h\left(y_{0}\right), B / \lambda\right) .
$$

Then, since $F_{1} \subset U_{1}$ and $F_{2} \subset U_{2}$, we have

$$
\bmod \left(F_{1}, F_{2} ; X\right) \leqslant \bmod \left(U_{1}, U_{2} ; X\right) .
$$

Let $k \geqslant 2$ be the largest integer such that

$$
B \geqslant 2^{k} \lambda^{2} A
$$

and denote

$$
A_{j}=\bar{B}_{d}\left(h\left(y_{0}\right), 2^{j} \lambda A\right) \backslash B_{d}\left(h\left(y_{0}\right), 2^{j-1} \lambda A\right), \quad j=1, \ldots, k .
$$

The WMDM-condition and the doubling property of $\mu$ then guarantee that for every $\gamma \in \Gamma\left(U_{1}, U_{2} ; X\right)$ the length in $(X, q)$ of the restriction of $\gamma$ to $A_{j}$ is at least

$$
\frac{\mu\left(B_{d}\left(h\left(y_{0}\right), 2^{j} \lambda A\right)\right)^{1 / 2}}{C_{W} C_{D}} .
$$

It follows that $\rho: U_{2} \backslash U_{1} \rightarrow[0, \infty]$,

$$
\rho(w)=\frac{1}{k} \sum_{j=1}^{k} \frac{C_{W} C_{D} \chi_{A_{j}}(w)}{\mu\left(B_{d}\left(h\left(y_{0}\right), 2^{j} \lambda A\right)\right)^{1 / 2}}
$$

is admissible for $\Gamma\left(U_{1}, U_{2} ; X\right)$. Integrating and applying (5), this yields

$$
\begin{aligned}
\bmod \left(U_{1}, U_{2} ; X\right) & \leqslant \frac{C_{W}^{2} C_{D}^{2}}{k^{2}} \sum_{j=1}^{k} \frac{\mathcal{H}_{q}^{2}\left(A_{j}\right)}{\mu\left(B_{d}\left(h\left(y_{0}\right), 2^{j} \lambda A\right)\right)} \\
& \leqslant \frac{2 \pi C_{W}^{2} C_{S}^{2} C_{D}^{6}}{k}
\end{aligned}
$$


Finally, combining Proposition 5.1, Proposition 6.1, and (35), we get

$$
k \leqslant \frac{2 \pi^{3} C_{W}^{2} C_{S}^{2} C_{D}^{6}(10 M)^{M}(M+2)^{2}}{\alpha^{M}},
$$

where $\alpha$ is the constant in Proposition 6.1. In particular, we have the desired bound for the ratio $B / A$. The proof is complete.

Acknowledgement. We thank the referee for several helpful comments.

\section{REFERENCES}

[1] M. Bonk. Uniformization of Sierpiński carpets in the plane. Invent. Math., 186(3):559-665, 2011.

[2] M. Bonk and B. Kleiner. Quasisymmetric parametrizations of two-dimensional metric spheres. Invent. Math., 150(1):127-183, 2002.

[3] M. Bonk and D. Meyer. Expanding Thurston maps, volume 225 of Mathematical Surveys and Monographs. American Mathematical Society, Providence, RI, 2017.

[4] G. David and S. Semmes. Strong $A_{\infty}$ weights, Sobolev inequalities and quasiconformal mappings. In Analysis and partial differential equations, volume 122 of Lecture Notes in Pure and Appl. Math., pages 101-111. Dekker, New York, 1990.

[5] J. Heinonen. Lectures on analysis on metric spaces. Universitext. SpringerVerlag, New York, 2001.

[6] T. Ikonen. Uniformization of metric surfaces using isothermal coordinates. Ann. Acad. Sci. Fenn. Math., to appear.

[7] A. Lohvansuu, K. Rajala, and M. Rasimus. Quasispheres and metric doubling measures. Proc. Amer. Math. Soc., 146(7):2973-2984, 2018.

[8] S. Merenkov and K. Wildrick. Quasisymmetric Koebe uniformization. Rev. Mat. Iberoam., 29(3):859-909, 2013.

[9] M. H. A. Newman. Elements of the topology of plane sets of points. Second edition, reprinted. Cambridge University Press, New York, 1961.

[10] K. Rajala. Uniformization of two-dimensional metric surfaces. Invent. Math., 207(3):1301-1375, 2017.

[11] M. Romney. Quasiconformal parametrization of metric surfaces with small dilatation. Indiana Univ. Math. J., 68(3):1003-1011, 2019.

[12] J. Väisälä. Lectures on n-dimensional quasiconformal mappings. Lecture Notes in Mathematics, Vol. 229. Springer-Verlag, Berlin-New York, 1971.

Department of Mathematics and Statistics, University of Jyväskylä, P.O. Box 35 (MaD), FI-40014, University of Jyväskylä, Finland.

\section{E-mail: Kai Rajala: kai.i.rajala@jyu.fi Martti Rasimus: martti.rasimus@gmail.com}

